The ITER Organization and domestic agencies together employ 2,000 people. Changing how ITER is managed will alter its culture. I aim to foster an atmosphere in which each party or individual feels personally responsible for the whole project, not just their area of competence. One of my first actions after becoming director was to address the staff of each domestic agency. The most striking moment was in a video session with all four Asian agencies. For the first time, colleagues in Japan, India, South Korea and China saw the faces of their counterparts, changing the dynamic towards a shared global ambition.

I am also implementing a new type of mobility throughout the project. This will enable appropriate domestic-agency staff to be temporarily seconded to the ITER site, or central-team staff to be assigned to domestic agencies.

The ITER Council has agreed to this new organization. I am grateful for their strong support and the progress already made in solving technical issues and improving communication.

\section{DISCRETIONARY FUND}

There is still much more to do. Authority requires the financial means to exercise it. I have asked for the creation of a reserve fund, to be put at my disposal. Each domestic agency will contribute, allowing me to take quick and efficient decisions to address issues as they arise. Terms of reference will be presented to the council in June for approval. The money will be drawn from the contributions of the ITER members in proportion to the amount they pay in.

In my experience of industrial projects, a reserve fund must comprise about $20 \%$ of

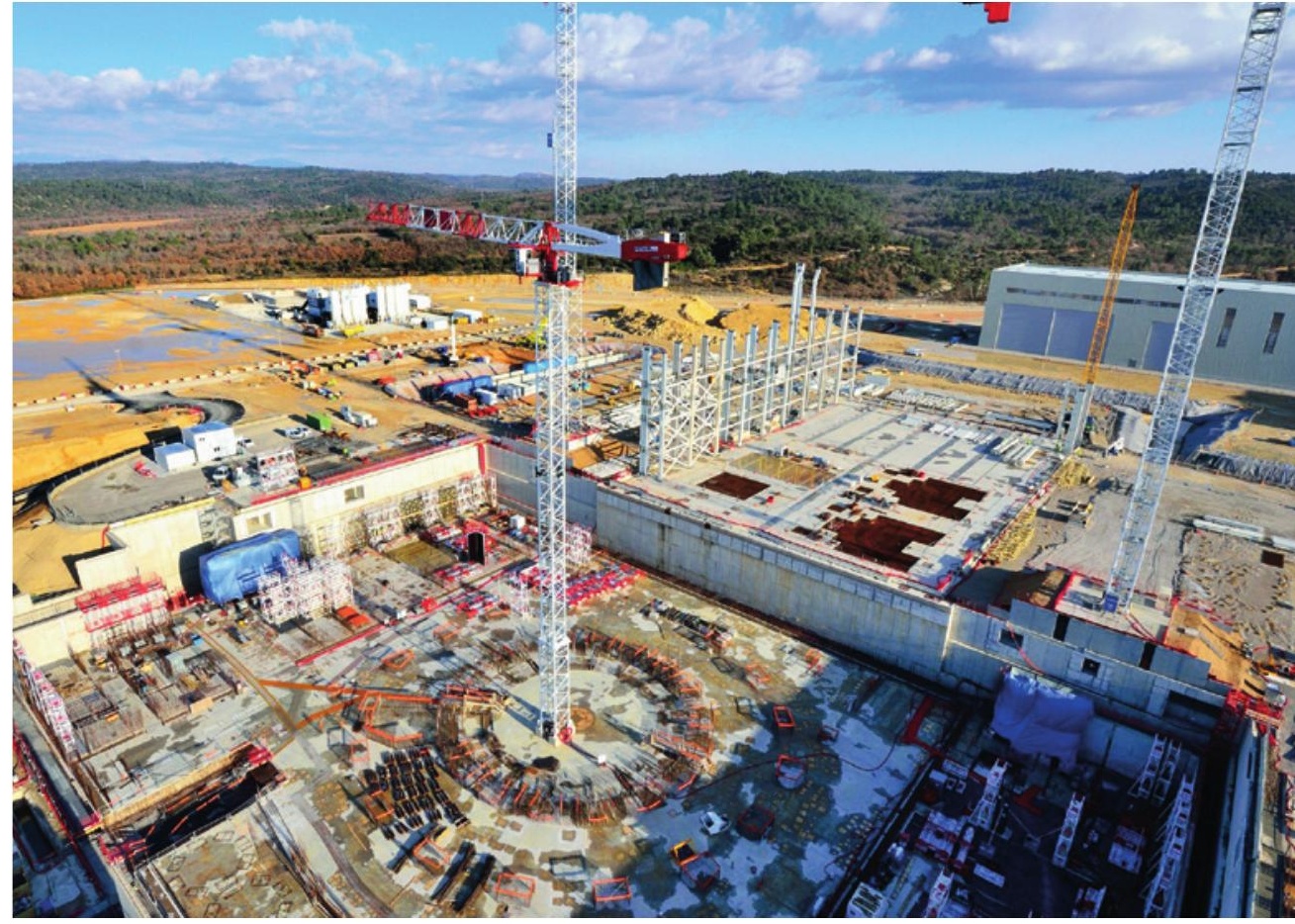

Construction at St-Paul-lez-Durance, France, site of the ITER nuclear-fusion experiment.

fabrication costs over the duration of construction. In my view, it was naive not to establish such a fund much earlier in ITER's history.

Before the end of this year, I am expected to submit, along with all stakeholders, an updated, robust and reliable schedule to the ITER Council, and a cost and risk analysis. With renewed management and a streamlined organization, we are now ready to prepare for the assembly and commissioning phase, the step before fusion switches on.
ITER will meet these challenges if it has the unanimous political support of the seven members, on the basis of the long-term value of fusion technology.

All of us at ITER have a huge, historic responsibility. The project may be the last chance we have this century to demonstrate that fusion is manageable.

Bernard Bigot is director-general of the ITER Organization, St-Paul-lez-Durance, France.

e-mail:bernard.bigot@iter.org

\title{
Use mouse biobanks or lose them
}

\section{Now that genetic engineering of mice is so easy, centralized repositories are essential, argue Kent Lloyd and colleagues.}

$\mathrm{T}$ ens of thousands of genetically engineered mice have been bred to probe human biology and disease. Their numbers are poised to mushroom. New genome-editing technologies such as CRISPR/Cas9 mean that making an animal that carries several customized mutations can be done in a matter of months, rather than years. Investigators who would not previously have considered making mutant mice are now doing so.

But laboratories that can make genetically modified mice are often unable to maintain them. Progeny frequently carry pathogens, lose carefully designed mutations or have other characteristics that confound experiments. So the mice that a researcher might dutifully ship to a colleague can be very different from those described in a paper. In 2013, the Mutant Mouse Resource and Research Centers (MMRRCs), a consortium of the US National Institutes of Health, found that 32 of around 200 mouse lines deposited with them from individual labs did not match researchers' descriptions. It is no wonder that many preclinical studies performed using mice are not reproducible ${ }^{1}$. 
As administrators of publicly funded animal repositories charged with preserving and distributing genetically engineered mouse lines, we routinely encounter - and correct - problems introduced by inappropriate breeding, animal husbandry and quality control. We worry that the explosion of new mouse models could create a surge of wasted effort and irreproducible results. Better use of repositories could avoid this problem.

\section{IN THE BANK}

For the past 16 years, the MMRRCs have maintained unique mouse models deposited by individual scientists. The collections encompass around 4,600 specific mutations in mice, and tens of thousands of mutations in frozen embryonic stem cells that can be used to generate mice. Last year, the MMRRCs and the Jackson Laboratory (JAX), a US non-profit biomedical-research organization, together distributed more than 200,000 live engineered mice, as well as frozen embryos and sperm representing hundreds of mutant lines. Australia, Europe and Japan also have government-funded repositories.

Mouse lines created by individual labs are often lost because of lack of interest or expertise. Commercial suppliers maintain only those lines that are in high demand. Making mouse lines publicly available from repositories renders these resources more accessible and eliminates costly, redundant efforts. It also relieves scientists of having to house animals and manage their breeding.

But fewer than half of the roughly 43,000 specific mutations listed in Mouse Genome Informatics, an international database of engineered mice, are listed as available from repositories. This is despite the fact that researchers funded by the US government are strongly encouraged to deposit mice for public distribution. A wide-ranging 2005 survey conducted by the NIH to investigate the extent of the problem found that, of 4,848 published mouse lines, only $12 \%$ were readily available from repositories. This forced scientists to rebuild mice; 2,655 had been remade at least once, and 702 had been remade independently more than three times (see 'Remaking mice'). The survey also spurred the Knockout Mouse Project (KOMP), which with international
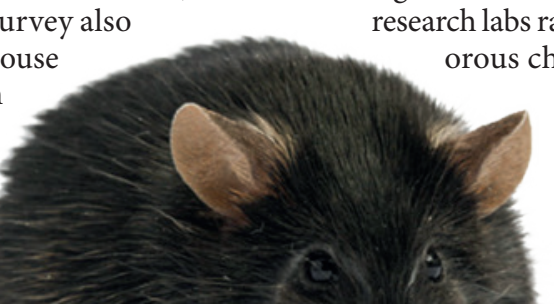

\section{REMAKING MICE}

When engineered animals are unavailable, researchers make them again. The most recent comprehensive survey, carried out in 2005 , found that researchers had made thousands of mouse lines more than once, wasting animals, time and money.

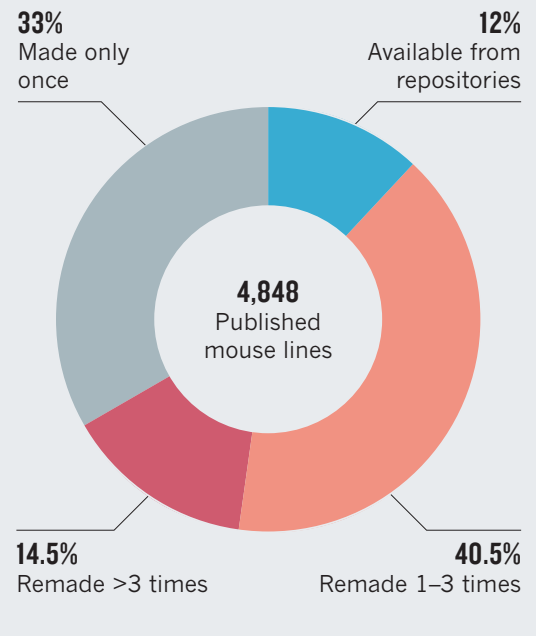

partners has made around 15,000 knockout alleles in embryonic stem cells, all deposited in repositories, including the KOMP Repository.

Since the survey, journals and funders have become more strict about requiring depositions. Yet we estimate that fewer than $20 \%$ of mouse lines are submitted. The rate is likely to fall as more scientists are able to engineer lines.

\section{QUALITY CONTROL}

Most researchers who use mice are experts in their fields rather than in mouse genetics, husbandry or pathology. Reagents remain relatively constant; a mouse is a living, breeding creature. Change is the default, and change over generations must be understood, monitored and managed.

Ordering a breeding pair of mice from a repository typically takes $3-5$ weeks and costs US $\$ 400-600$, more if mice must be created from frozen stocks. (Engineering a mouse from scratch can cost upwards of $\$ 20,000$.) Many researchers prefer to get animals straight from a colleague. Although sharing is laudable, mice obtained from research labs rarely go through the rigorous checks that are standard practice in repositories. Receiving labs are risking the reliability of subsequent experiments - and perhaps even the health of their vivaria.

Repositories ensure the quality and welfare of distributed animals and supply expertise to guide reliable studies.
This means that researchers learn more from the animal experiments they conduct. They address the problem of improperly identified animals in several ways. By the time results from an engineered mouse line are published in a paper, the line has probably bred through several generations and undergone genetic drift. Repositories can accept a mouse line on article submission (and even hold off distribution until publication) and maintain animals closer to the original description.

What is more, repositories routinely analyse animals' genomes before making them available and so catch mistakes. Sometimes researchers overlook mutations that have been engineered into a mouse line, which can alter the animals' traits or corrupt attempts at appropriate breeding. In 2013, genetic tests on 416 mutant mouse lines submitted to JAX and the MMRRCs found that $15 \%$ carried mutations for traits other than that specified, or contained genetic markers used to track mouse breeding not intended to be part of the line. The most frequent mixup is essentially a typo: a common strain annotated as C57BL/6J is instead another called C57BL/6N. Although the pups look identical, the mice are very different. The $6 \mathrm{~N}$ mice quickly develop bad eyesight, and 6J mice are susceptible to diabetes and obesity. Such traits can cause results to be misinterpreted and experiments to be irreproducible.

Researchers might also treat a line as breeding pure (with no mixing of genetic backgrounds) when it does not. All MMRRCs have received submissions of

"Repositories
ensure the
quality and
welfare of
distributed
animals."
engineered lines that contained a mixture of mice carrying the mutation and 'wild-type' mice. We have also encountered many instances in which a genetic marker (such as a fluorescent protein) was decoupled from the mutation it was supposed to identify. No surprise, then, that researchers who receive mice from colleagues can conduct several rounds of experiments, only to find that they have been studying mice that lack the desired mutation.

Another underappreciated source of variability is microbes. Identical mutations in a gene active in T cells made at two institutions revealed similarities on the molecular and cellular levels, but profound differences in the animals. At one institution, mice consistently developed prolapsed rectums and died two months after birth. Careful investigation revealed that this was caused by a stealth outbreak of the bacterium Helicobacter hepaticus ${ }^{2}$. Repositories control for pathogens through frequent monitoring - and the ability to revive the strain under germ-free conditions from frozen embryos or gametes.

The microbiome (the collective DNA 


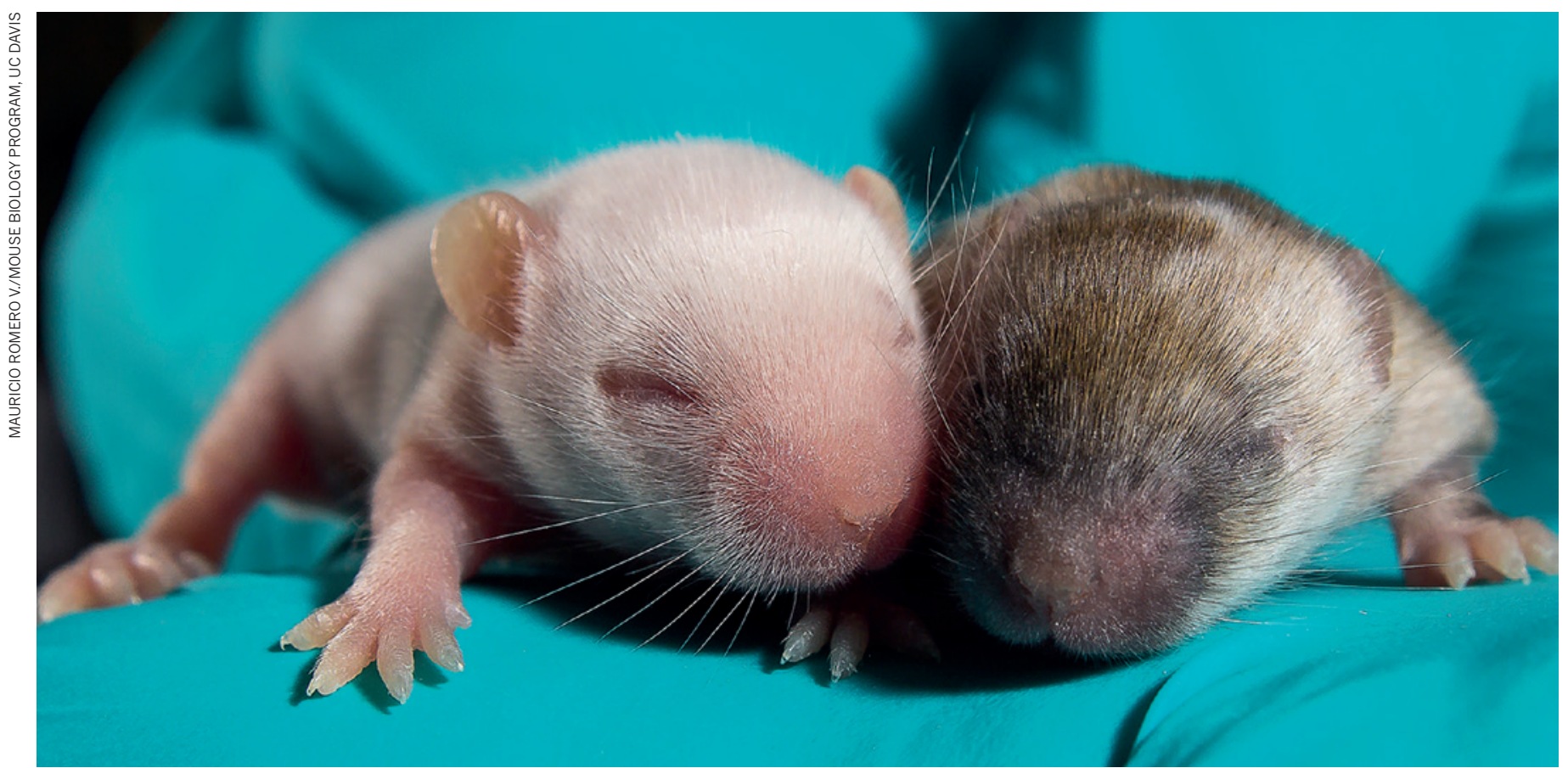

Littermates are often a mixture of mice carrying a required mutation and 'wild type'.

of microbes residing in the gut), which can vary for mice at different institutions even when they are fed identical diets, also causes surprising differences. Repositories are beginning to use DNA sequencing to define common variables such as diet, housing and other factors that may modulate microbiota ${ }^{3}$. This could reveal the effects of these variables on a variety of mouse traits, and make animal studies conducted by collaborating investigators more efficient and reproducible.

Finally, repositories can help to pin down unexpected causes of mouse traits, making the mice more useful to researchers. For example, a strong difference between two strains' responses to cocaine and methamphetamine was recently mapped to a site in a single gene that differed between the strains ${ }^{4}$. At the MMRRC at the University of North Carolina at Chapel Hill, work to sort out crosses between mouse lines generated a variety of traits, including a line of mice that developed severe inflammation in the gut. The line has now been distributed to several organizations as a model for the human disease known as spontaneous colitis ${ }^{5}$.

\section{FOUR STEPS FORWARD}

If these benefits are to accrue, researchers must deposit their mice in repositories. Currently they may not for three reasons: they are unaware that repositories exist; they mistakenly think that they must pay for submission; or they want greater control over when and how their lines are distributed. Scientists should be better global citizens, and funders and journals must be more diligent in creating and enforcing requirements for deposition.
Next, scientists must use mice from repositories. The catalogue number and other documentation that repositories supply will ensure clear tracking of the mice, and the quality control that repositories routinely perform will ensure that descriptions match the actual mouse. The ARRIVE (Animals in Research: Reporting In Vivo Experiments) guidelines ${ }^{6}$, increasingly followed by publications that report animal research, should be amended to encourage acquisition from repositories. Mice obtained from non-specialists should undergo documented quality control - from genotyping to pathogen monitoring.

Third, repositories must work together to enhance their services. They should organize themselves into an integrated global network to share best practices, harmonize protocols and procedures, and innovate. Goals should include the development of certified qualitycontrol practices and streamlining institutional transfer agreements to take in new mouse strains and to guarantee better distribution, particularly across international borders.

Continued investment is all the more important as researchers are called on to meet government mandates that may require individual studies to include more animals. Recent examples include Research Councils UK's requirement for researchers to statistically validate the numbers of mice used and the NIH's mandate to study both male and female mice ${ }^{7}$. Meanwhile, more mouse lines are being made by individual labs, and the International Mouse Phenotyping Consortium is set to complete more than 20,000 mouse models by 2021, encompassing virtually the entire mouse genome.

Without sufficient investment, we fear a vicious cycle in which repositories are unable to cope with increasing demands and become less able to serve the scientific community, keeping fewer live mice ready for distribution. Worse, they will close. (At least one already has.)

Like money in the bank, repositories keep mouse models safe, secure and available for withdrawal. Just as a bank makes returns on investments, repositories add scientific value and utility to deposited mouse lines: they increase reliability through curation, preservation, genetic quality control, protection from pathogens and more.

Kent Lloyd is director of the Mutant Mouse Resource and Research Center (MMRRC) at the University of California, Davis, California, USA. Craig Franklin is director of the MMRRC at the University of Missouri, Columbia, Missouri, USA. Cat Lutz is director of the MMRRC at the Jackson Laboratory in Bar Harbor, Maine, USA. Terry Magnuson is director of the MMRRC at the University of North Carolina at Chapel Hill, North Carolina, USA. e-mail:kclloyd@ucdavis.edu

1. Perrin, S. A. Nature 507, 423-425 (2014).

2. Franklin, C. L. ILAR J 47, 140-154 (2006).

3. Ericsson, A. C. et al. PLOS ONE 10, e0116704 (2015).

4. Kumar, V. et al. Science 342, 1508-1512 (2013)

5. Rogala, A. R. et al. Mamm. Genome 25, 95-108 (2014).

6. Kilkenny, C., Browne, W. J., Cuthill, I. C., Emerson, M. \& Altman, D. G. PLoS Biol. 8, e1000412 (2010).

7. Clayton, J. A. \& Collins, F. S. Nature 509, 282-283 (2014). 